\title{
Local Research Ethics Committees can audit ethical standards in research
}

\author{
Jonathan Berry The Princess Royal Hospital, Haywards Heath, West Sussex
}

\begin{abstract}
Objectives - To show that a Local Research Ethics Committee (LREC) can carry out an audit of ethical standards in research. To find out if a researcher met certain ethical standards in recruiting subjects for clinical trials and in obtaining their consent.

Design - Postal questionnaire.

Setting - Clinical research by one doctor during one year.

Subjects - Eleven patients entered in clinical trials. Main outcome measures - Success in ethics committee obtaining data. Achievement of ethical standards in recruitment of subjects and in obtaining consent.

Results - The audit was successfully carried out and standards were partly met.

Conclusions - Local Research Ethics Committees can carry out audits of the conduct of research projects which they have approved. Provision for possible audits can be made at the time of application to the committee. Our committee thought the ethical standards in the research which we audited were acceptable.
\end{abstract}

\section{Introduction}

Research Ethics Committees have a duty to ensure that good ethical research is carried out. Both subjects and researchers need the protection of external ethical review. Smith has recently called for the medical profession to tackle research misconduct effectively. ${ }^{1}$ I show here that LRECs can monitor ethical standards in research and incidentally help prevent misconduct.

Local Research Ethics Committees in the United Kingdom are advisory. They have guidance from the Department of Health but no rules as to how they should operate. ${ }^{2}$ Our committee decided that we might want to monitor ethical standards in local research. We prepared by telling researchers on our application form that we would sometimes contact subjects for this purpose (appendix a). We ask researchers to agree to this at the time they apply for LREC approval and also to obtain subjects' consent to the release of their contact details to the committee as part of their consent to the study.

We noticed a doctor who was doing more commercially sponsored research than his peers. Such trials are usually closely monitored by the sponsors who review the trial records and patient notes. We decided to ask the patients if some ethical standards had been met.

\section{Methods}

The committee authorized the chairman to ask the doctor for the names and addresses of all the patients he had entered in three clinical trials in the previous year. The doctor replied in ten days with 11 namesce. and addresses. I sent the subjects a letter (appendix b), but no reminders, explaining that the committee was making a routine check on standards in research and enclosing a questionnaire (appendix c).

\section{Results}

There were ten replies over the following two months with nine completed questionnaires. One relative wrote saying that the subject was confused and unable to complete it. All nine responding patients knew they were research subjects. They all correctly identified the illness which was being treated in the trial (five hypertension, two indigestion, one depression) except one being treated for depression who chose "I'd rather not say". Two did not recall being given an information sheet but all who did understood it. Eight patients thought the researcher had answered all their questions and one was not sure. Seven subjects thought they had had enough time to consider entering the trial, one thought he had not and one was not sure. Eight subjects understood that they could have the usual treatment if they didn't want to enter the trial and one was unsure. Three subjects said they had not been told what to do if the medicine made them worse. None was offered money or gifts to enter a trial.

\section{Discussion}

The purpose of our audit was firstly to find out if 
such an audit could be done and secondly to make sure that ethical standards were being maintained. This is the first time we have conducted an audit in this way. I found no published account of similar work done by an ethics committee. Pharmaceutical and research companies closely monitor researchers' records but their interest is mainly in the validity of the data. We used a simple questionnaire focusing on the things we thought were important, particularly the validity of the subjects' consent. The issues which concerned us were:

1) Did the patients know that they were research subjects?

2) Had they been given enough information and enough time to give valid consent?

3) Had they been told what to do if there was a problem?

This usually requires an information sheet, including contact details of the researcher, an explicit offer of standard treatment if the subject chooses not to enter the trial, all the subject's questions answered satisfactorily and enough time to consider.

Inclusion of the response "I'd rather not say" for each question indicated that subjects did not have to answer all the questions and that this response was acceptable. It was only chosen once by a patient being treated for depression who chose not to indicate the illness for which he was being treated in the trial.

Our first objective was achieved as it was an easy task to conduct the audit. It was done with no specific funding but if we do it regularly there will be resource implications. This could be built into the charges which are levied by many LRECs.

We did not know what standard to set. Ideally there should be $100 \%$ compliance but in the absence of published data we used this audit to help us set standards for future audits. The committee felt the results in this small study indicated an adequate standard of ethical practice. In reaching this conclusion we made allowance for the interval between recruitment and our questionnaire, which could have resulted in inaccurate recall of events. We wrote to the doctor telling him the summarised anonymous results and our conclusion. We also emphasised that we expect all patients to be given an information sheet and enough time to consider and that they must understand clearly what to do in the event of an adverse reaction. We have not sent the results to the subjects but have sent each a letter of thanks saying that the committee was satisfied that the research was done correctly.

Audits need to be conducted with careful attention to technique. Discovery of the subjects' details from the researcher is a potential source of inaccuracy. $\mathrm{He} /$ she might choose not to disclose all of the subjects or he/she might try to prompt them in their responses to the questionnaire. We did nothing to stop the subjects approaching the researcher for advice on how to complete the questionnaire. Therem was no suggestion of impropriety in this study. If $\overrightarrow{\overline{\bar{D}}}$. audits of this kind become more widely used, better? ways of discovering and questioning the subjects will $\vec{F}$ become established. Researchers might be required ? to send lists of subjects routinely to LRECs and? questionnaires could be administered in person. $\frac{\bar{\sigma}}{\overline{0}}$ Researchers who know that their research may be $\overrightarrow{\mathbb{\Phi}}$ audited by an ethics committee will take even greater care to maintain standards.

There is a continuing debate about whether it is $\overrightarrow{0}$ good for subjects to know that research has beenapproved by an LREC. ${ }^{3}$ We had to reveal the exis- ${ }_{-}$ tence of the LREC to obtain the subjects' consent to disclosure of contact details but there was no need to mention that the LREC had approved the research.

With increasing awareness of fraud and malprac-io tice in research, monitoring of this kind is necessary. $\omega$ Audit of ethical standards in research can be done by research ethics committees if arrangements are made in advance. If committees are to have credibility they $\vec{\square}$ must be able to ensure that the standards which they approve in theory are being applied in practice. This $\Phi$ is at least as important as LRECs auditing their own processes and structures.

\section{Acknowledgements}

I am grateful to the other members of the Downs East Local Research Ethics Committee for their advice and encouragement and to the doctorg for cooperating fully and agreeing to publication.

fonathan Berry, $M B, \quad B S, \quad M R C P, \quad F R C R$, is $\frac{\vec{\circ}}{3}$ Chairman of Mid Downs East Local Research Ethics Committee and a Radiologist in the Diagnostic Imaging Department, The Princess Royal Hospital, Lewes Road, Haywards Heath.

Appendix a) Extract from our committee's application form for ethical approval.

"From time to time the committee will approach을 subjects to audit ethical aspects of the research. The $>$ committee asks that this possibility is included in the을. patient information sheet thus: 'This research has been approved by our Local Research Ethics ${ }^{\circ}$ Committee. The committee sometimes asks us for ${ }^{N}$ the names and addresses of research subjects to N check that research is being done correctly. Byo agreeing to take part in the research you also agreeo that the researcher can tell the committee your name $\frac{\mathbb{\Phi}}{}$ and address but you don't have to answer the com- $\stackrel{?}{+}$ mittee's questions if you don't want to.'

"Would you be willing to identify research subjects to the committee for this purpose?"

Appendix $b$ ) Letter sent to research subjects

"I am writing as chairman of the Local Research 
Ethics Committee to ask for your help. The committee's job is to make sure that medical research is done in the correct way. Dr X has told us that you agreed to help him with some research sometime in the last two years. We would be most grateful if you would complete the enclosed questionnaire about it. I would like to emphasise that this is a routine inquiry that we make from time to time. We do it to make sure that standards are kept high. We have no reason to think that there is anything wrong.

"Please telephone me if you have any questions."

\section{Appendix c) Questionnaire}

"Please answer the questions by putting a circle round your answer

1) Before you had this letter did you know that you were taking part in research?

Yes No Not sure I'd rather not say

2) When the doctor asked you to help with the research what illness or symptom was the treatment for?

Diabetes High Blood Pressure Indigestion Chest infection Depression Not sure

I'd rather not say

3) Were you given an information sheet?

Yes No Not sure I'd rather not say

4) If you were given an information sheet did you understand it?

Yes No Not sure I'd rather not say
5) Did the doctor answer all your questions?

Yes No Not sure I'd rather not say

6) Did you have enough time to think about it before agreeing to take part?

Yes No Not sure I'd rather not say

7) Did you realize that you didn't have to take part if you didn't want to and that the doctor would have given you the normal treatment?

Yes No Not sure I'd rather not say

8) Did the doctor tell you what to do if the medicine made you worse?

Yes No Not sure I'd rather not say

9) Were you offered any gifts or money?

Yes No Not sure I'd rather not say

Please write here anything you want to tell us about being in medical research. Thank you for your help."

\section{References}

1 Smith R. Time to face up to research misconduct. British Medical fournal 1996; 312: 789-90.

2 Department of Health. Local Research Ethics Committees. London: Department of Health. Undated but published in August 1991 as health service guideline HSG(91)5.

3 Sheffield Institute of Biotechnological Law and Ethics. Is it ethical to inform potential participants in research of LREC approval of research protocols? Sheffield: Sheffield Institute of Biotechnological Law and Ethics, 1996. 